\title{
Nonmodular Tapered Fluted Titanium Stems Osseointegrate Reliably at Short Term in Revision THAs
}

\author{
Nemandra A. Sandiford MBBS, MSc, FRCS(Tr\&Orth), \\ Donald S. Garbuz MD, MHSc, FRCS(C), Bassam A. Masri MD, FRCS(C), \\ Clive P. Duncan MB, MSc, FRCS(C)
}

Received: 7 February 2016/Accepted: 13 September 2016/Published online: 26 September 2016

(C) The Association of Bone and Joint Surgeons \& 2016

\begin{abstract}
Background The ideal femoral component for revision THA is undecided. Cylindrical nonmodular stems have been associated with stress shielding, whereas junctional fractures have been reported with tapered fluted modular titanium stems. We have used a tapered fluted nonmodular titanium femoral component (Wagner Self-locking [SL] femoral stem) to mitigate this risk. This component has been used extensively in Europe by its designer surgeons, but to our knowledge, it has not been studied in North
\end{abstract}

Each author certifies that he or a member of his immediate family, has no funding or commercial associations (eg, consultancies, stock ownership, equity interest, patent/licensing arrangements, etc) that might pose a conflict of interest in connection with the submitted article.

All ICMJE Conflict of Interest Forms for authors and Clinical Orthopaedics and Related Research ${ }^{\mathbb{B}}$ editors and board members are on file with the publication and can be viewed on request.

Clinical Orthopaedics and Related Research ${ }^{\mathbb{R}}$ neither advocates nor endorses the use of any treatment, drug, or device. Readers are encouraged to always seek additional information, including FDAapproval status, of any drug or device prior to clinical use.

Each author certifies that his institution approved the human protocol for this investigation, that all investigations were conducted in conformity with ethical principles of research, and that informed consent for participation in the study was obtained.

This work was performed at the Faculty of Medicine, University of British Columbia (Vancouver, BC, Canada).

N. A. Sandiford ( $\bowtie)$, D. S. Garbuz

Division of Reconstructive Orthopaedics (Hip and Knee), Faculty of Medicine, University of British Columbia, 3rd Floor 910 West 10th Avenue, Vancouver, BC V5Z 4E3, Canada e-mail: nemsandiford@hotmail.com

B. A. Masri, C. P. Duncan

Department of Orthopaedics, Faculty of Medicine, University of British Columbia, Vancouver, BC, Canada
America. Added to this, the design of the component has changed since early reports.

Questions/purposes We asked: (1) Does the Wagner SL stem have low rates of rerevision and other complications at a minimum 2 years after surgery? (2) Is the Wagner SL stem associated with high levels of patient function and pain relief at a minimum 2 years after surgery? (3) Does the Wagner SL stem have low rates of subsidence at a minimum 2 years after surgery? (4) Is the Wagner SL stem associated with proximal femoral bone remodeling at a minimum 2 years after surgery?

Method Between May 2011 and December 2012, we performed 198 femoral revisions, of which 104 (53\%) were performed using the Wagner SL femoral stem; during that period, our institution gradually shifted toward increasing use of these stems for all but the most severe revisions, in which modular fluted stems and proximal femoral replacements still are used on an occasional basis. Median followup in this retrospective study was 32 months (range, 24-46 months), and one patient was lost to followup before the 2-year minimum. The femoral deformities in this series were Paprosky Type I (10 hips), Paprosky Type II (26), Paprosky Type IIIA (52), Paprosky Type IIIB (nine), and Paprosky Type IV (two). Functional assessment was performed using the Oxford Hip Score (OHS), WOMAC, SF12, and the University of California Los Angeles (UCLA) activity score. All complications and cases of revision were documented. All patients had radiographs performed within 1 year of the latest followup. These were assessed by two surgeons for signs of proximal femoral bone remodeling and subsidence.

Results Complete preoperative scores were available for 98 patients (98 of 104; 94\%). The mean OHS preoperatively and at final followup were 39 (SD, 15) and 87 (SD, 19), respectively ( $<<0.001$; mean difference, $48 ; 95 \% \mathrm{CI}$, 
43-53). Average WOMAC scores were 44 (SD, 15) and 87 (SD, 20), respectively ( $<<0.001$; mean difference, 43 ; 95\% CI, 38-48). At final followup, signs of restoration of proximal femoral bone stock was noted in 45 of 103 hips (44\%). Six (six of 104; 6\%) patients had subsidence of 10 $\mathrm{mm}$ to $15 \mathrm{~mm}$. In the remainder (98 of $104 ; 94 \%$ ), the mean subsidence was $2 \mathrm{~mm}$ (range, $0-9 \mathrm{~mm}$ ). One revision was performed for loosening associated with infection.

Conclusions The Wagner SL stem is a viable option for patients with Paprosky Types II and III defects undergoing revision THA. This component provides high levels of patient function with low revision rates and low rates of subsidence during the early postoperative phase. They provide a viable alternative to modular components for treatment of Types II and III defects without the risk of junctional fractures. They can be used for very selected Type IV defects, however this extent of bone loss is most easily addressed with other techniques such as a proximal femoral replacement.

Level of Evidence Level IV, therapeutic study

\section{Introduction}

Femoral component revision, especially in the presence of substantial bone loss affecting the proximal femur, can be technically challenging $[19,21]$. Fully porous-coated cylindrical stems have provided encouraging clinical results [3, 15, 19, 22, 24]. However substantial proximal femoral stress shielding leading to loss of femoral bone stock and anterior thigh pain have occurred with these designs, resulting in a cautious approach to their ongoing use [27]. Modular tapered fluted titanium stems have been associated with encouraging results as reported by numerous authors, leading to their increasing popularity in North America [4, 5, 10, 11, 14, 23, 29, 32, 33]. Furthermore, a comparison of the chrome-cobalt extensively coated stems and tapered titanium stems in one study suggested superiority of the latter in terms of the clinical outcome [26]. However, these modular stems have drawbacks. Concerns arose at our institution regarding the modularity of the tapered titanium stems after several junctional fractures were noted and were reported by other authors $[4,7]$. This has led to efforts to refine the fatigue strength of the modular stem junction with the introduction of newer stem designs. However, medium-term results of these efforts are lacking. In an effort to mitigate the potential risk of stem fracture, we began using the nonmodular tapered fluted design in 2011.

We believed that the nonmodular design would show results comparable to published results with modular stems $[23,28,30,31,33]$. Several studies $[6,14,20]$ support this view, however some of the medium- to long-term studies $[6,14]$ reflect use of early designs of this prosthesis. In addition, some of these studies represent the efforts of surgeons involved in the design of the implants used $[34,35]$. The aim of our study is to present the clinical, radiologic, and functional results from a single center, with revision THAs performed by surgeons who were not designers of the implant, who used the most recent iteration of a nonmodular stem design, and with patients having a minimum followup of 2 years.

We therefore asked: (1) Do repeat revision rates with the Wagner Self-Locking (SL) stem (Wagner SL Revision ${ }^{\circledR}$ hip stem; Zimmer Inc, Warsaw, IN, USA) match published results for this and other implants? (2) Does the Wagner SL stem provide comparable or acceptable patient function and pain relief at a minimum of 2 years? (3) Is subsidence with the Wagner SL stem a clinical or functional problem at a minimum 2 years after surgery? (4) Does proximal femoral remodeling occur?

\section{Patients and Methods}

Between May 2011 and December 2012, 198 revision THAs were performed at our institution. Of those, 104 $(53 \%)$ were performed using the Wagner SL stem. During that period this implant was used predominantly to treat Paprosky Types II and III defects. Modular fluted stems were still used for complex cases where we expected that a monoblock would not have the required versatility but a segmental replacement was not necessary, whereas various types were used in the remainder. Fifty-five men and 49 women underwent revision THA between May 2011 and December 2012. The median age of this cohort was 72 years (range, 40-94 years). Median duration of followup was 31 months (range, 24-46 months). One patient was lost to followup; otherwise, the outcomes of all patients are known. The median BMI was $30 \mathrm{~kg} / \mathrm{m}^{2}$ (range, $18-40 \mathrm{~kg}$ / $\mathrm{m}^{2}$ ). Patients had a median of three procedures before their most recent revision. Fifty-two percent of our cohort were classified as being in Charnley Class C. The median American Society of Anesthesiologists (ASA) score [29] was 2 (range, 1-4) (Table 1). The main indications for revision included aseptic loosening (48\%), infection (24\%), and periprosthetic fracture (15\%) (Table 2). Sixty-three patients had femoral bone loss that was categorized as severe: 52 defects were categorized as Paprosky Type IIIA; nine were Type IIIB defects; and two were Type IV defects (Table 1). Acetabular revision was performed in 98 hips. Trilogy ${ }^{\circledR}$ acetabular components (Zimmer) were used in $30 \%$ (31 cases) of patients, trabecular metal modular cups were used in $64 \%$ (67 cases), and the acetabular component was unrevised in $6 \%$ (six cases). Seven patients died of 
Table 1. Patient demographics

\begin{tabular}{lc}
\hline Demographic & Variable \\
\hline Number of patients & 104 \\
Mean age (years) & 69 \\
Mean followup (months) & 31 \\
Mean BMI $\left(\mathrm{kg} / \mathrm{m}^{2}\right)$ & 29 \\
Mean number of previous revision surgeries & 3 \\
Charnley class & 16 \\
A & 34 \\
B & 54 \\
C & \\
ASA class & 9 \\
1 & 37 \\
2 & 57 \\
3 & 1 \\
4 & \\
Paprosky type & 10 \\
I & 31 \\
II & 52 \\
IIIA & 9 \\
IV & 2 \\
\hline ASA American Society of Anesthesiologiss. & \\
\hline
\end{tabular}

ASA = American Society of Anesthesiologists.

Table 2. Indications for revision in the patient cohort

\begin{tabular}{lc}
\hline Indication & Number $(\%)$ \\
\hline Aseptic loosening & $50(48)$ \\
Infection & $25(24)$ \\
Periprosthetic fracture & $16(15)$ \\
Instability & $7(7)$ \\
Fractured stem & $2(2)$ \\
Pseudotumor & $2(2)$ \\
Conversion of fusion & $1(1)$ \\
Anterior thigh pain from previous primary stem & $1(1)$ \\
\hline
\end{tabular}

unrelated causes. The results of these patients have been excluded. The outcome of each femoral component up to the point of death of the patients is known. These patients were pain-free up to their last followup. There were no revisions or pending revisions in this group, and no femoral component revisions or pending revisions up to their final followup.

The Wagner SL Revision ${ }^{\circledR}$ stem is a nonmodular tapered fluted titanium stem. It is manufactured from a titanium-aluminum-niobium (Ti AlNb) alloy and has a gritblasted surface to facilitate bone ongrowth. The stem has a $2^{\circ}$-taper and eight longitudinal cutting flutes which engage the endosteal bone, providing rotational stability. It is inserted after preparation of the femur with conical reamers. This "cone within a cone" fit provides axial stability. It has a circular cross-section, which allows the surgeon to optimize or "dial in" the version of the femoral neck.

\section{Operative Technique}

All procedures were performed through an extensile posterior approach. An extended trochanteric osteotomy was performed in 41 cases (39\%) to expedite removal of wellfixed cemented or cementless stems and in cases of varus remodeling of the proximal femur to facilitate safe "straight shot" access to the diaphyseal canal. These were planned during the preoperative templating stage. The stem size, length, and zone of fixation were not affected by the extended trochanteric osteotomy. Cerclage wires or cables were used for fixation of all osteotomies. We did not change our preoperative plan for any patient who had an extended trochanteric osteotomy. The femur was handreamed with incremental conical reamers. The stem size and orientation and the head and neck sizes were fine-tuned with the use of a modular trial. The diameter of the final reamer used matched the sizes of the stem used. Uncemented acetabular components were used in all cases in which the acetabulum was revised.

Weightbearing was based on the severity of the preoperative bone loss and security of the reconstruction, but was partial for the first 6 weeks for all patients and was advanced based on the clinical progress and radiographic findings with time.

Patients were reviewed preoperatively, at 3 months and 1 year after surgery, and then annually thereafter. Clinical and radiographic assessments were performed at each visit.

Clinical outcome was recorded using a standardized quality-of-life (QOL) assessment tool, which included the Oxford Hip Score (OHS) [20], WOMAC score [3], the SF12 [36], and the University of California Los Angeles (UCLA) activity score [1] preoperatively and at the latest followup. Patients also were stratified according to their level of comorbidity using the ASA [29] and Charnley classification systems [9]. The WOMAC and SF-12 scores were converted to scores out of 100 , where higher scores indicate better function. All radiographs were assessed by at least two of three surgeons (NS, CPD, DSG) for signs of implant stability as described by Regis et al. [25] and for evidence of stem subsidence. Stem subsidence was measured using the distance from the tip of the greater trochanter to the shoulder of the prosthesis as described by Callaghan et al. [8]. Remodeling of the proximal femoral bone stock was assessed using the system proposed by Böhm and Bischel [6]. Subsidence was considered to be clinically significant if it exceeded $10 \mathrm{~mm}$ [20]. 
Heterotopic ossification was classified according to the system of Brooker et al. [7]. Proximal femoral bone deficiencies were classified according to the system of Weeden and Paprosky [38]. All complications were recorded. Failure was defined as revision or impending revision of the stem for any reason.

\section{Statistical Analysis}

The median for QOL and hip function in this cohort were calculated using SAS ${ }^{\circledR}$ Version 9.3 (SAS Institute Inc, Cary, NC, USA).

\section{Results}

At a minimum of 2 years, no hips had been revised for aseptic loosening. One revision of the stem and cup had been performed for infection, and two acetabular revisions had been performed for instability. Dislocation occurred in five patients, but only two required revision surgery. Two patients required additional surgery in the form of irrigation and débridement of wound hematomata at 4 and 5 weeks after the index procedure. Complications encountered included five dislocations (five of $104 ; 5 \%$ ) and three cases of infection (three of 104; 3\%) (Table 3). Grade I heterotopic ossification was noted on 21 (20\%) radiographs, Grade II on eight (8\%), and Grade III on three $(3 \%)$. There were no radiographs showing progressive radiolucent lines suggestive of aseptic loosening or loss of proximal femoral bone stock suggestive of stress shielding at the most recent followup.

Complete preoperative scores were available for 98 patients (98 of 104; 94\%), and we noted improvement in pain and function. The mean OHS preoperatively and at final followup were 39 (SD, 15) and 87 (SD, 19), respectively ( $\mathrm{p}<0.001$; mean difference, 48; 95\% CI, 43-53). Average WOMAC scores were 44 (SD, 15) and 87 (SD,

Table 3. Complications in the study patients

\begin{tabular}{ll}
\hline Complication & Number \\
\hline Subsidence (>10 mm) & 6 \\
Dislocation & 5 \\
Anterior thigh pain & 1 \\
Return to operating room (irrigation and débridement) & 2 \\
Infection & 3 \\
Scar revision & 1 \\
Intraoperative fracture & 1 \\
Extended trochanteric osteotomy nonunion & 1 \\
\hline
\end{tabular}

20 ), respectively ( $\mathrm{p}<0.001$; mean difference, $43 ; 95 \% \mathrm{CI}$, $38-48$ ). Mean pre- and postoperative scores for the mental component of the SF-12 were $44(\mathrm{SD}, 11)$ and 46 (SD, 10), respectively ( $\mathrm{p}=0.172$; mean difference, $2 ; 95 \% \mathrm{CI},-1$ to $5)$. Average pre- and postoperative scores for the physical component of the SF-12 scores were 35 (SD, 11) and 51 (SD, 11), respectively ( $\mathrm{p}<0.001$; mean difference, 16 ; 95\% CI, 14-18). The average UCLA score preoperatively was $3(\mathrm{SD}, 1)$, and at final followup it was $5(\mathrm{SD}, 2)$ ( $\mathrm{p}<$ 0.001; mean difference, 2; 95\% CI, 1.6-2.4). Twenty-four percent of patients (25 of 104) had a UCLA score of 7 or more, reflecting a moderate to high activity level.

Median subsidence was $2 \mathrm{~mm}$ (range, $2-15 \mathrm{~mm}$ ). Subsidence was observed between 3 and 6 months in all cases. Six $(6 \%)$ patients had subsidence greater than $10 \mathrm{~mm}$ (median, $12 \mathrm{~mm}$; range, 10-15 mm). These components stabilized by the 1-year followup. No patient in this subset of six had symptoms suggesting stem loosening.

The median pre- and postoperative OHS in this group (with subsidence $>10 \mathrm{~mm}$ ) were 40 (range, 15-80) and 85 (range, 80-100), respectively. The difference of medians was $45(\mathrm{p}=0.002)$. Median pre- and postoperative WOMAC scores were 47 (range, 29-90) and 88 (range, 75-100), respectively. The difference of medians was 41 (p $=0.003$ ). Median pre- and postoperative UCLA scores were 3 (range 1-3) and 6 (range, 1-9). The difference of medians was $3(\mathrm{p}=0.008)$. Femoral deficiencies in this group were Paprosky Type IIIA (five patients) and Type IIIB (one patient). At most recent followup the final median OHS in the group with subsidence was 85 (range, 79-100) compared with 84 (range, 79-100) in the entire cohort. The difference of medians was 1 ( $\mathrm{p}=0.802 ; 95 \%$ CI, -17 to 13 ). Final median WOMAC scores were 84 (range, 75-100) and 86 (range, 79-100), respectively. The difference between the medians was $2(\mathrm{p}=0.905 ; 95 \% \mathrm{CI}$, -18 to 18$)$. The latest UCLA scores in the group with subsidence and the entire cohort were 6 (range, 1-9) and 7 (range, 1-9), respectively. Difference between the medians was 1 ( $\mathrm{p}=0.236 ; 95 \% \mathrm{CI},-3$ to 1$)$.

Signs of proximal femoral bone remodeling were observed from as early as 3 months after surgery. At most recent followup, signs of restoration of proximal femoral bone stock were seen in 45 patients $(47 \%)$. We observed no evidence of progressive bone loss or disuse osteopenia in any patient; these are common observations after use of chrome-cobalt extensively porous-coated stems. Forty extended trochanteric osteotomies had progressed to full union by the final followup. There was nonunion of an extended trochanteric osteotomy in one patient. An additional corrective osteotomy through the medial cortex was performed in 15 patients (14\%) to address severe varus remodeling. All of these patients achieved union. 


\section{Discussion}

Management of femoral bone loss in revision THA can be challenging. Fully coated cylindrical cobalt-chrome stems have been associated with proximal femoral stress shielding and loss of bone stock [24, 26]. Obtaining fixation in patients with large defects (Paprosky Types IIIB and IV) can be difficult with this design [17]. Numerous authors have reported encouraging clinical and radiologic outcomes with the use of tapered fluted modular titanium stems [22, 23, 26, 30, 31, 38, 39], however, observation of junctional fractures at the modular stems [26] led to increased interest in the use of the nonmodular Wagner SL tapered fluted titanium stem at our institution. We therefore asked: (1) Do repeat revision rates with the Wagner SL stem match published results for this and other implants? (2) Does the Wagner SL stem provide comparable or acceptable patient function and pain relief at a minimum of 2 years? (3) Is subsidence a clinical or functional problem at a minimum of 2 years after surgery? (4) Does remodeling of the proximal femur occur?

This study has several limitations mainly owing to the relatively short duration of followup. Most patients appeared to do well in the short term; however, subsidence of 10 to $15 \mathrm{~mm}$ was observed in six patients $(6 \%)$. This group was considered to be at risk for loosening and instability and followed closely for a longer time. Our study is retrospective, and we acknowledge selection bias. The stems in question were used in only 53\% (104 of 198) of patients, and more complicated hips, such as those with Paprosky Type IV defects and segmental bone loss, were treated with proximal femoral replacements. Modular stems and extensively coated stems were used in $21 \%$ of patients (22 of 104). Although the use of these rapidly declined during the study period as they were replaced by the monoblock design, we have used modular stems, extensively porous-coated stems, and others with encouraging results $[6,12,14,16,23,26,32]$. Thus, it is possible that patients treated with modular designs would have done as well.

One patient (one of $104 ; 1 \%$ ) underwent a two-stage revision for infection 9 months after surgery. No rerevisions were performed for aseptic loosening or progressive subsidence in contrast to reported rerevision rates of $5 \%$ to $8 \%$ [2, 6, 14, 25]. The most common complications reported with the Wagner SL stem (other than subsidence) have been dislocations and periprosthetic fractures $[6,14,25]$. Dislocation rates of $5.7 \%$ to $13.9 \%$ have been reported with the first- and second-generation designs of the Wagner SL stem [6, 14, 25]. Five of our patients $(5 \%)$ experienced dislocations. Two patients underwent revision of the acetabular component to a constrained liner and three were treated successfully with closed reduction. Both patients undergoing reoperation had severe abductor deficiency. Three patients had diagnoses of deep periprosthetic joint infection; two of these patients were treated without surgery with long-term antibiotics. One patient (one of 104; $1 \%$ ) underwent a two-stage revision for infection 9 months after surgery. None of our patients experienced a periprosthetic fracture. Three patients required reoperations at this early stage, which is comparable to the incidence reported by other authors for complications including infection, dislocation, and subsidence [6, 14, 25].

Although our patients had substantial comorbidities, we noted significant early improvement in pain levels and overall function, as measured with validated patient-reported outcomes. Similar improvements in functional outcome have been reported by other authors in short-, medium- and long-term studies. Bohm and Bischel [6] reported significant improvement in pain, function, and ROM at an average of 4.8 years after surgery with this prosthesis. Gutiérrez Del Alamo et al. [14] found similar results at a mean 8.4 years in a group of older patients. Regis et al. [25] and Weber et al. [37] reported Harris hip scores of 75 and 76 at 13.9 and 5.5 years, respectively, with use of the Wagner SL stem in revision cases. Thirty percent of our patients were employed full-time at the time of final followup. The results of our study also suggest that encouraging results can be achieved by a cross section of surgeons and not simply specialist units.

During the first year, six of our patients (six of 104; 6\%) had radiologic subsidence of $10 \mathrm{~mm}$ or greater (average, 12 $\mathrm{mm}$; range, 10-15 $\mathrm{mm}$ ), which stabilized during the first year. The outcome scores of this small group were not lower than those in the overall cohort. Regis et al. [25], Gutiérrez Del Alamo et al. [14], and Bohm and Bischel [6]

Table 4. Comparison of rates of clinically significant subsidence and dislocations

\begin{tabular}{|c|c|c|c|c|c|}
\hline Study & Number of patients & Followup (months) & Mean age of patients (years) & Subsidence $>10 \mathrm{~mm}$ & Dislocation \\
\hline Regis et al. [25] & 41 & 167 & 61 & $8(20 \%)$ & $4(10 \%)$ \\
\hline Böhm and Bischel [6] & 129 & 97 & 70 & $26(20 \%)$ & $7(5 \%)$ \\
\hline Gutiérrez Del Alamo et al. [14] & 79 & 101 & 72 & $15(19 \%)$ & $11(14 \%)$ \\
\hline Hartwig et al. [16] & 37 & 27 & 64 & $7(19 \%)$ & None \\
\hline Current study & 104 & 31 & 69 & $6(6 \%)$ & $5(5 \%)$ \\
\hline
\end{tabular}


reported incidences of subsidence greater than $10 \mathrm{~mm}$ of $20 \%, 19 \%$, and $20 \%$, respectively. Revision for progressive subsidence in their patients, however, were two of 68 $(2.9 \%)$, one of $79(1.3 \%)$, and one of $123(0.8 \%)$, respectively. Baktir et al. [2] supported these established studies with an $11 \%$ incidence of subsidence greater than $10 \mathrm{~mm}$. These studies [2, 6, 14, 25] reflect results of the early design of the Wagner SL stem, suggesting that the design change potentially affects subsidence. Early subsidence has been reported to be a predictor of early revision attributable to aseptic loosening [18], however we did not experience this in our cohort, in keeping with Girard et al. [13], who reviewed 183 revisions at 5.9 years followup and found that, in contrast to proximal fit and fill stems, early subsidence did not affect the function or osseointegration of conical revision stems. The results of our study are comparable to the best in the literature (Table 4). Subsidence has been associated with component undersizing [6]. We were able to avoid this in our current series.

Böhm and Bischel [6] and others [5, 14, 25] have described improvements in the quantity and quality of proximal femoral bone stock associated with the use of the Wagner SL stem. Our observations are similar. Fifty-two percent of our patients had Paprosky Type III defects $(48 \%$ with Type IIIA, 5\% with Type IIIB), yet bony reconstitution was noted in $47 \%$ of this group. No proximal femoral bone loss (stress shielding) was noted. An extended trochanteric osteotomy was used in 41 patients (39\%). Forty of these $(98 \%)$ patients achieved complete union in keeping with findings by others [6, 14, 25]. Bony union was observed at 6 months after surgery. Heterotopic ossification was observed in 31 patients. This rate of bone remodeling and healing is likely the result of the elastic modulus of the stem being relatively similar to bone.

The results of our study suggest that encouraging levels of patient function and low complication and revision rates can be achieved with a nonmodular tapered titanium stem in patients with Paprosky Types II and III bone defects at a minimum of 2 years after surgery along with proximal femoral bone regeneration. These results are comparable to those of prior studies by the surgeon designers of this implant [34, 35]. They suggest that tapered fluted nonmodular stems are a viable option for treatment of patients with bone deficiency and can successfully mitigate the risk of junctional fractures which is a potential risk with modular designs. Modular designs still have a role, particularly in patients with very high offsets, and segmental replacement remains the preferred treatment for severe segmental bone deficiency. The incidence of subsidence in our patients is lower than for patients in previous studies with this design, however further prospective randomized controlled trials are needed to confirm this finding. Further research also is needed to establish the medium- and longterm results of Wagner SL nonmodular design.

\section{References}

1. Amstutz HC, Thomas BJ, Jinnah R, Kim W, Grogan T, Yale C. Treatment of primary osteoarthritis of the hip: a comparison of total joint and surface replacement arthroplasty. $J$ Bone Joint Surg Am. 1984;66:228-241

2. Baktır A, Karaaslan F, Gencer K, Karaoğlu S. Femoral revision using the Wagner SL Revision stem: a single-surgeon experience featuring 11-19 years of follow-up. J Arthroplasty. 2015;30:827834

3. Bellamy N, Buchanan WW, Goldsmith $\mathrm{CH}$, Campbell J, Stitt LW. Validation study of WOMAC: a health status instrument for measuring clinically important patient relevant outcomes to antirheumatic drug therapy in patients with osteoarthritis of the hip or knee. J Rheumatol. 1988;15:1833-1840.

4. Berry DJ. Femoral revision: distal fixation with fluted, tapered grit-blasted stems. J Arthroplasty. 2002;17(4 suppl 1):142-146.

5. Bircher HP, Riede U, Lüem M, Ochsner PE. [The value of the Wagner SL revision prosthesis for bridging large femoral defects][in German]. Orthopade. 2001;30:294-303.

6. Böhm P, Bischel O. Femoral revision with the Wagner SL revision stem: evaluation of one hundred and twenty-nine revisions followed for a mean of 4.8 years. J Bone Joint Surg Am. 2001;83:1023-1031.

7. Brooker AF, Bowerman JW, Robinson RA, Riley LH Jr. Ectopic ossification following total hip replacement: incidence and a method of classification. J Bone Joint Surg Am. 1973;55:1629-1632.

8. Callaghan JJ, Salvati EA, Pellicci PM, Wilson PD Jr, Ranawat CS. Results of revision for mechanical failure after cemented total hip replacement, 1979 to 1982: a two to five-year follow-up. J Bone Joint Surg Am. 1985;67:1074-1085.

9. Charnley J. The long-term results of low-friction arthroplasty of the hip performed as a primary intervention. J Bone Joint Surg Br. 1972;54:61-76.

10. Dawson J, Fitzpatrick R, Carr A, Murray D. Questionnaire on the perceptions of patients about total hip replacement. J Bone Joint Surg Br. 1996;78:185-190.

11. Fink B, Urbansky K, Schuster P. Mid term results with the curved modular tapered, fluted titanium Revitan stem in revision hip replacement. Bone Joint J. 2014;96:889-895.

12. Franzén H, Mjöberg B, Önnerfält R. Early loosening of femoral components after cemented revision: a roentgen stereophotogrammetric study. J Bone Joint Surg Br. 1992;74:721-724; erratum in J Bone Joint Surg Br. 1993;75:169.

13. Girard J, Roche O, Wavreille G, Canovas F, Le Béguec P. Stem subsidence after total hip revision: 183 cases at 5.9 years followup. Orthop Traumatol Surg Res 2011;97:121-126

14. Gutiérrez Del Alamo J, Garcia-Cimbrelo E, Castellanos V, GilGaray E. Radiographic bone regeneration and clinical outcome with the Wagner SL revision stem: a 5-year to 12-year follow-up study. J Arthroplasty. 2007;22:515-524.

15. Hamilton WG, Cashen DV, Ho H, Hopper RH Jr, Engh CA. Extensively porous-coated stems for femoral revision: a choice for all seasons. J Arthroplasty. 2007;22(4 suppl 1):106-110.

16. Hartwig CH, Böhm P, Czech U, Reize P, Küsswetter W. The Wagner revision stem in alloarthroplasty of the hip. Arch Orthop Trauma Surg. 1996;115:5-9.

17. Heyligers IC, Schreurs BW, van Haaren EH. Femoral revision with impaction bone grafting and a cemented polished tapered stem. Oper Orthop Traumatol. 2014;26:156-161. 
18. Kärrholm J, Malchau H, Snorrason F, Herberts P. Micromotion of femoral stems in total hip arthroplasty: a randomized study of cemented, hydroxyapatite-coated, and porous-coated stems with roentgen stereophotogrammetric analysis. J Bone Joint Surg Am. 1994;76:1692-1705

19. Kavanagh BF, Ilstrup DM, Fitzgerald RH Jr. Revision total hip arthroplasty. J Bone Joint Surg Am. 1985;67:517-526.

20. Kolstad K, Adalberth G, Mallmin H, Milbrink J, Sahlstedt B. The Wagner revision stem for severe osteolysis: 31 hips followed for 1.5-5 years. Acta Orthop Scand. 1996;67:541-544.

21. Muirhead-Allwood S, Sandiford NA, Skinner JA, Hua J, Muirhead W, Kabir C, Walker PS. Uncemented computer-assisted design-computer-assisted manufacture femoral components in revision total hip replacement: a minimum follow-up of ten years. J Bone Joint Surg Br. 2010;92:1370-1375.

22. Munro JT, Garbuz DS, Masri BA, Duncan CP. Role and results of tapered fluted modular titanium stems in revision total hip arthroplasty. J Bone Joint Surg Br. 2012;94(11 suppl A):58-60.

23. Munro JT, Masri BA, Garbuz DS, Duncan CP. Tapered fluted modular titanium stems in the management of Vancouver B2 and B3 peri-prosthetic fractures. Bone Joint J. 2013;95(11 suppl A):17-20.

24. Paprosky WG, Greidanus NV, Antoniou J. Minimum 10-yearresults of extensively porous-coated stems in revision hip arthroplasty. Clin Orthop Relat Res. 1999;369:230- 242.

25. Regis D, Sandri A, Bonetti I, Braggion M, Bartolozzi P. Femoral revision with the Wagner tapered stem: a ten- to 15-year followup study. J Bone Joint Surg Br. 2011;93:1320-1326.

26. Richards CJ, Duncan CP, Masri BA, Garbuz DS. Femoral revision hip arthroplasty: a comparison of two stem designs. Clin Orthop Relat Res. 2010;468:491-496.

27. Roberson JR. Proximal femoral bone loss after total hip arthroplasty. Orthop Clin North Am. 1992;23:291-302.

28. Rodriguez JA, Deshmukh AJ, Robinson J, Cornell CN, Rasquinha VJ, Ranawat AS, Ranawat CS. Reproducible fixation with a tapered, fluted, modular, titanium stem in revision hip arthroplasty at 8-15 years follow-up. J Arthroplasty. 2014;29(9 suppl):214-218.

29. Saklad M. "Grading of patients for surgical procedures". Anesthesiology. 1941;2: 281-284.

30. Sandiford NA, Duncan CP, Garbuz DS, Masri BA. Tapered, fluted titanium stems in revision total hip arthroplasty: role and results in contemporary practice. Instr Course Lect. 2015;64:359366.

31. Sculco PK, Abdel MP, Lewallen DG. Management of femoral bone loss in revision total hip arthroplasty. Hip Int. 2015;25:380 387.

32. Sporer SM, Paprosky WG. Femoral fixation in the face of considerable bone loss: the use of modular stems. Clin Orthop Relat Res. 2004:429:227-231.

33. Van Houwelingen AP, Duncan CP, Masri BA, Greidanus NV, Garbuz DS. High survival of modular tapered stems for proximal femoral bone defects at 5 to 10 years followup. Clin Orthop Relat Res. 2013;471:454-462.

34. Wagner H, Wagner M. Cone prosthesis for the hip joint. Arch Orthop Trauma Surg. 2000;120:88-95.

35. Wagner H, Wagner M. Conus hip prosthesis. Acta Chir Orthop Traumatol Cech 2001;68:213-221

36. Ware J, Kosinski M, Keller SD. A 12-item Short-Form Health Survey: construction of scales and preliminary tests of reliability and validity. Med Care. 1996;34:220-233.

37. Weber M, Hempfing A, Orler R, Ganz R. Femoral revision using the Wagner stem: results at 2-9 years. Int Orthop. 2002;26:36-39.

38. Weeden SH, Paprosky WG. Minimal 11-year follow-up of extensively porous-coated stems in femoral revision total hip arthroplasty. J Arthroplasty. 2002;17(4 suppl 1):134-137.

39. Wirtz DC, Gravius S, Ascherl R, Thorweihe M, Forst R, Noeth U, Maus UM, Wimmer MD, Zeiler G, Deml MC. Uncemented femoral revision arthroplasty using a modular tapered, fluted titanium stem: 5- to 16-year results of 163 cases. Acta Orthop. 2014;85:562-569. 